Pacific Journal of Mathematics

APPROXIMATIONS OF FUNCTIONS ON THE INTEGERS D JOSEPH SI PERT UHLAN 


\title{
APPROXIMATION OF FUNCTIONS ON THE INTEGERS
}

\author{
Gene F. Rose and Joseph S. Ullian
}

How can algorithms be used to analyze nonrecursive functions? This question motivates the present work.

Let us suppose that a particular function, with natural numbers as arguments and values, is known to be completely defined but not recursive. Then by Church's thesis, ${ }^{1}$ no algorithm gives the functional value for every argument. In some practical situation, however, where a particular sequence of arguments is of interest, it might suffice to have an "approximating algorithm" that performs as follows when applied to the successive arguments in the sequence: for each argument, the algorithm computes a number; for some arguments, this number may differ from the actual functional value, but after sufficiently many arguments have been processed, the proportion of such cases never exceeds a prescribed real number less than unity. If such an approximating algorithm exists whenever the given sequence of arguments is infinite, nonrepeating and effectively generable, then the given function is in some (conceivably useful) sense susceptible to analysis by mechanical means. Functions of this last kind are the object of our investigation; when the above notions are made precise in $\S 1$, they are called "recursively approximable" functions.

In $\S 2$ it is shown that uncountably many nonrecursive functions are recursively approximable; in $\S 3$, that uncountably many functions are not recursively approximable. ${ }^{2}$

1. A number-theoretic notion of approximation. Given any function $f$, any partial function $\varphi,{ }^{3}$ and any sequence $x_{0}, x_{1}, \cdots$ of natural numbers, let "err $(n)$ " denote the number of natural numbers $i<n$ such that $f\left(x_{i}\right) \neq \varphi\left(x_{i}\right)$. If $E$ is a real number and, for all sufficiently large $n$, $\operatorname{err}(n) / n \leqq E$, then we say that $\varphi$ approximates

Received December 5, 1962.

1 Cf [3].

2 An analogous notion of approximable function, involving finite sets of arguments rather than sequences, is considered in [5], where a function is called " $m$-in- $n$-computable" if there is an algorithm that produces at least $m$ correct functional values for every set of $n$ arguments. It was shown that uncountably many functions are not $m$-in- $n$ computable for any $m>0$. The existence of nonrecursive $m$-in- $n$-computable functions with $m>0$ was left an open question; an affirmative answer, however, was soon provided by Dana Scott in an unpublished communication.

${ }^{3}$ By "function" we mean, unless otherwise specified, "total singulary function" (in the sense of $[\mathbf{1}]$ p. xxi). A "partial function" is any singulary function whose domain is a subset of the natural numbers. 
$f$ with error $E$ on the given sequence.

It may happen that, for every infinite nonrepeating recursive enumeration $x_{0}, x_{1}, \cdots,{ }^{5}$ there is a partial recursive function $\varphi$ that approximates $f$ with error $E$ on $x_{0}, x_{1}, \cdots .{ }^{6}$ In this case we say that $f$ is recursively approximable with error $E$. A function recursively approximable with some error $<1$ is called recursively approximable.

2. Recursively approximable functions. Are there recursively approximable functions other than the recursive functions? The MyhillFriedberg notion of maximal set provides an affirmative answer through Theorem 2.1. ${ }^{7}$ By Corollary 2.2 below, every recursion function $f$ recursively approximates the uncountably many functions which agree with $f$ on a maximal set. ${ }^{8}$ In fact, we establish a stronger result as follows.

We consider an extension of the notion of maximal set. For convenience, a set $C$ is called cohesive if it is infinite and, for every recursively enumerable set $R$, either $R \cap C$ or $\bar{R} \cap C$ is finite. A set is quasi-maximal if for some positive natural number $m$, its complement is the union of $m$ cohesive sets. Thus the maximal sets are those quasi-maximal sets for which the number $m$ can be taken as 1 . Through Theorem 2.1, the notion of quasi-maximal set provides a sufficient condition that a function $f$ be recursively approximable. This condition is that there exist a recursive function $r$ that agrees on some quasimaximal set with $f$.

THEOREM 2.1. Let $f$ be any function, $r$ any recursive function, $Q$ any quasi-maximal set such that $f$ and $r$ agree on $Q$. Then $r$ recursively approximates $f$ with arbitrary positive error on every infinite nonrepeating recursive enumeration.

Proof. Assume that $\bar{Q}=C_{1} \cup \cdots \cup C_{m}$ where the $C$ 's are cohesive sets. Let $E$ be any positive real number and $x_{0}, x_{1}, \cdots$ any infinite nonrepeating recursive enumeration. Choose a natural number $p \geqq$ $(m+1) / E$ and, for each natural number $j<p$, let $X_{j}=\left\{x_{i} \mid i \equiv j \bmod p\right\}$.

${ }^{4}$ In order to realize an approximating algorithm in the sense of the Introduction, it would be necessary to require that $\varphi$ be defined for all $x_{i}$. It will be obvious, however, that the current results would be unaffected by this additional requirement.

5 A "recursive enumeration" is any sequence $x(0), x(1), \cdots$ where $x$ is a recursive function.

${ }^{6}$ Terminology regarding recursive functions and recursively enumerable sets is essentially that of [3]. However, "recursive" is used throughout for "general recursive," and the empty set is regarded as recursively enumerable.

7 A set $\boldsymbol{M}$ is maximal if (i) $\bar{M}$ is infinite and (ii) for every recursively enumerable set $R$, either $R \cap \bar{M}$ or $\bar{R} \cap \bar{M}$ is finite. The existence of recursively enumerable maximal sets is established in [2].

${ }^{8}$ Functions $r$ and $f$ are said to agree on a set $X$ if, for all $x \in X, r(x)=f(x)$. 
For each $k(1 \leqq k \leqq m)$ we consider two cases.

Case 1. $\left\{x_{0}, x_{1}, \cdots\right\} \cap C_{k}$ is finite. Lat $q_{k}$ be the number of its members. Then the number of numbers $i<n$ such that $f\left(x_{i}\right) \neq r\left(x_{i}\right)$ and $x_{i} \in C_{k}$ is $\leqq q_{k}$.

Case 2. $\left\{x_{0}, x_{1}, \cdots\right\} \cap C_{k}$ is infinite. Then for some $j<p, X_{j} \cap C_{k}$ is infinite. Because $C_{k}$ is cohesive and $X_{j}$ is recursively enumerable, $\bar{X}_{j} \cap C_{k}$ is finite; let $q_{k}$ be the number of its members. Now $\left\{x_{i} \mid f\left(x_{i}\right) \neq\right.$ $r\left(x_{i}\right)$ and $\left.x_{i} \in C_{k}\right\} \subset C_{k}=\left(X_{j} \cap C_{k}\right) \cup\left(\bar{X}_{j} \cap C_{k}\right) \subset X_{j} \cup\left(\bar{X}_{j} \cap C_{k}\right)$. Therefore the number of numbers $i<n$ such that $f\left(x_{i}\right) \neq r\left(x_{i}\right)$ and $x_{i} \in C_{k}$ is $\leqq((n-1) / p)+1+q_{k}$.

By hypothesis, if $f\left(x_{i}\right) \neq r\left(x_{i}\right)$ then $x_{i} \in C_{1} \cup \cdots \cup C_{m}$. Hence $\operatorname{err}(n) \leqq$ $m(n-1) / p+m+q_{1}+\cdots+q_{m}$. Therefore

$$
\operatorname{err}(n) / n \leqq m / p+\left(p\left(q_{1}+\cdots+q_{m}\right)+m p-m\right) /(n p)
$$

and, for all $n \geqq p\left(q_{1}+\cdots+q_{m}\right)+m p_{-}-m, \operatorname{err}(n) / n \leqq E$.

COROLLARY 2.2. For every recursive function $r$, there are uncountably many functions $f$ such that $r$ recursively approximates $f$ with arbitrary positive error on every infinite nonrepeating recursive enumeration.

Proof. Given any recursive function $r$, choose any quasi-maximal set $Q$. For each subset $S$ of $\bar{Q}$, let $f_{S}$ be the function such that $f_{S}(x)=1-r(x)$ if $x \in S, f_{S}(x)=r(x)$ otherwise. The functions $f_{S}$, being in one-to-one correspondence with the subsets of $\bar{Q}$, are uncountable.

For brevity, we will call a function "maximal" if it is not recursive and it agrees on some maximal set with some recursive function, "quasi-maximal" if it is not recursive and it agrees on some quasimaximal set with some recursive function. In Theorem 2.1, the quasimaximal functions were shown to be recursively approximable. By means of Theorems 2.5 and 2.6, we will show that there are uncountably many quasi-maximal functions, and consequently uncountably many recursively approximable functions, that are not maximal. For this purpose, let us define the rank of a quasi-maximal set $Q$ to be the minimum number $m$ such that $\bar{Q}$ is the union of $m$ cohesive sets. Then define the rank of a quasi-maximal function $f$ to be the minimum number $m$ such that $f$ agrees on some quasi-maximal set of rank $m$ with some recursive function. Thus the maximal sets (functions) are the quasi-maximal sets (functions) of rank 1.

LEMmA 2.3. If $C_{1}, \cdots C_{m}$ are cohesive sets, then every recursively enumerable subset of $C_{1} \cup \cdots \cup C_{m}$ is finite. 
Proof. Assume that $R$ is an infinite recursively enumerable subset of $C_{1} \cup \cdots \cup C_{m}$. Then there is a recursive function $r$ such that $r(0), r(1), \cdots$ enumerates $R$ without repetition. Let $R_{j}=\{r(i) \mid i \equiv$ $j \bmod m+1\}(j=0, \cdots, m)$. Then $R_{0}, \cdots, R_{m}$ are $m+1$ disjoint infinite recursively enumerable subsets of $C_{1} \cup \cdots \cup C_{m}$. Hence at least two distinct $R$ 's, say $R_{j}$ and $R_{k}$, have an infinite intersection with the same $C_{i}$. Since $R_{k} \subset \bar{R}_{j}$, it follows that $C_{i} \cap R_{j}$ and $C_{i}-R_{j}$ are infinite, contrary to the fact that $C_{i}$ is cohesive.

LEMmA 2.4. If $Q$ and $R$ are quasi-maximal sets and $R-Q$ is finite, then the rank of $Q \leqq$ rank of $R$.

Proof. Let $m$ be the rank of $Q, n$ the rank of $R$. There are cohesive sets $D_{1}, \cdots, D_{n}$ such that

$$
\bar{R}=D_{1} \cup \cdots \cup D_{n} \text {. }
$$

Then

$$
\bar{Q}=\left(D_{1}-Q\right) \cup \cdots \cup\left(D_{n}-Q\right) \cup(R-Q) .
$$

Since $\bar{Q}$ is infinite and $R-Q$ is finite, at least one $D_{i}-Q$ is infinite. We may assume without loss of generality that the infinite sets $D_{i}-Q$ are $D_{1}-Q, \cdots, D_{h}-Q$ where $1 \leqq h \leqq n$. Hence from (2.2)

$$
\bar{Q}=\left(\left(D_{1}-Q\right) \cup F\right) \cup \cdots \cup\left(\left(D_{h}-Q\right) \cup F\right)
$$

where $F$ is finite. For each $i(1 \leqq i \leqq h), D_{\imath}-Q$, being an infinite subset of the cohesive set $D_{i}$, is obviously cohesive, hence $\left(D_{i}-Q\right) \cup F$ is cohesive. Since $Q$ has rank $m$, it follows from (2.3) that $m \leqq h \leqq n$.

THeOREM 2.5. For every natural number $m>1$, there is a recursively enumerable quasi-maximal set of rank $m$. Hence there are infinitely many quasi-maximal sets that are not maximal.

Proof. Choose a recursively enumerable maximal set $Q_{1}$ and let $e$ be a recursive function such that $e(0), e(1), \cdots$ enumerates $Q_{1}$ without repetition. Define by induction on $m$ the sets $Q_{m}$ and $C_{m}(m=1,2, \cdots)$ thus.

$$
C_{1}=\bar{Q}_{1} ; \text { for all } m>1, Q_{m}=e\left(Q_{m-1}\right) \text { and } C_{m}=Q_{m-1}-Q_{m} \cdot{ }^{9}
$$

Clearly, each $Q_{m}$ is recursively enumerable. By induction on $m$ we establish the following properties of the $Q$ 's and $C$ 's. For all $m \geqq 1$,

$$
Q_{m+1} \subset Q_{m}
$$

${ }^{9}$ For any function $f$ and set $X$, we denote the image of $X$ under the mapping $f$ by " $f(X) . "$ 


$$
C_{m+1}=e\left(C_{m}\right)
$$

$$
C_{m} \text { is cohesive; }
$$

$$
\bar{Q}_{m}=C_{1} \cup \cdots \cup C_{m} .
$$

Basis. Let $m=1$. Now $Q_{2}=e\left(Q_{1}\right)$ and $Q_{1}=e(N)$ where $N$ is the set of all natural numbers. $Q_{1} \subset N$; therefore (2.5) holds. Next, note that $e$ is a one-to-one mapping. Hence $C_{2}=e(N)-e\left(Q_{1}\right)=$ $e\left(N-Q_{1}\right)=e\left(C_{1}\right)$; i.e. (2.6) holds. Because $Q_{1}$ is maximal, $C_{1}$ is cohesive; i.e. (2.7) holds. By (2.4), (2.8) holds.

Induction step. Let $m>1$. By (2.4), $Q_{m+1}=e\left(Q_{m}\right)$ and $Q_{m}=e\left(Q_{m-1}\right)$. By induction hypothesis, $Q_{m} \subset Q_{m-1}$. Therefore (2.5) holds. By (2.4), $C_{m+1}=e\left(Q_{m-1}\right)-e\left(Q_{m}\right)=e\left(Q_{m-1}-Q_{m}\right)=e\left(C_{m}\right)$; i.e. (2.6) holds. By induction hypothesis $C_{m-1}$ is cohesive, hence infinite. Then by (2.6) $C_{m}$ is infinite. Let $R$ be any recursively enumerable set. The set $\{x \mid e(x) \in R\}$ (call it $R^{\prime}$ ) is recursively enumerable. In view of (2.6) and the fact that $e$ is one-to-one, $C_{m} \cap R=e\left(C_{m-1} \cap R^{\prime}\right)$ and $C_{m}-R=$ $e\left(C_{m-1}-R^{\prime}\right)$. Suppose that $C_{m} \cap R$ is infinite. Then $C_{m-1} \cap R^{\prime}$ must be infinite. Then, because $C_{m-1}$ is cohesive, $C_{m-1}-R^{\prime}$ is finite, and consequently $C_{m}-R$ is finite. Thus (2.7) holds. Finally, in view of (2.5), $\bar{Q}_{m}=\bar{Q}_{m-1} \cup\left(Q_{m-1}-Q_{m}\right)=\bar{Q}_{m-1} \cup C_{m}$. Hence by induction hypothesis (2.8) holds.

Having established (2.5)-(2.8) we now show that, for all $m>1$, $Q_{m}$ has rank $m$. By (2.8) and (2.7), $Q_{m}$ ash rank $\leqq m$. Let $D_{1}, \cdots, D_{n}$ be any cohesive sets such that $\bar{Q}_{m}=D_{1} \cup \cdots \cup D_{n}$. By (2.8) each $C_{i}$ has an infinite intersection with at least one $D_{k}$. Moreover, if $1 \leqq$ $i<j \leqq m, C_{i}$ and $C_{j}$ cannot both have an infinite intersection with the same $D_{k}$. If they did, then by (2.8) $C_{i} \cap D_{k} \subset \bar{Q}_{i} \cap D_{k}$ and, by (2.4) and (2.5), $C_{j} \cap D_{k} \subset Q_{j-1} \cap D_{k} \subset Q_{i} \cap D_{k}$; then $Q_{i} \cap D_{k}$ and $\bar{Q}_{i} \cap D_{k}$ would both be infinite, contrary to the fact that $Q_{i}$ is recursively enumerable and $D_{k}$ is cohesive. Thus for each $i$ between 1 and $m$ there must be a distinct $k$ between 1 and $n$. Therefore $n \geqq m$. We conclude that $Q_{m}$ has rank $m$.

Theorem 2.6. For every natural number $m>1$, there are uncountably many quasi-maximal functions of rank $m$. Hence there are uncountably many quasi-maximal functions that are not maximal.

Proof. By Theorem 2.5 there is a recursively enumerable quasimaximal set $Q$ of rank $m$. For each of the uncountably many subsets $S$ of $\bar{Q}$ let $f_{S}$ be the function such that 


$$
f_{S}(x)=\left\{\begin{array}{l}
0 \text { if } x \in Q \\
1 \text { if } x \in S \\
2 \text { otherwise }
\end{array}\right.
$$

There are uncountably many functions $f_{S}$ since they are in one-to-one correspondence with the sets $S$. If $f_{S}$ were recursive, then $\bar{Q}$ would be the infinite recursively enumerable set $\left\{x \mid f_{S}(x) \neq 0\right\}$, contrary to Lemma 2.3. Hence each $f_{S}$ is nonrecursive. Therefore, since $f_{S}$ agrees on $Q$ with the constant function $0, f_{S}$ is a quasi-maximal function of rank $\leqq m$.

Moreover, consider any quasi-maximal set $R$ and any recursive function $r$ such that $f_{S}$ agrees on $R$ with $r$. Now $Q$ and $\{x \mid r(x) \neq 0\}$ are recursively enumerable and $\{x \mid r(x) \neq 0\} \cap Q \subset \bar{R}$. Hence $\{x \mid r(x) \neq 0\} \cap$ $Q$, being a recursively enumerable subset of $\bar{R}$, is finite by Lemma 2.3. Hence $\{x \mid r(x) \neq 0\}-Q$, which $=\{x \mid r(x) \neq 0\}-(\{x \mid r(x) \neq 0\} \cap Q)$, is a recursively enumerable subset of $\bar{Q}$. Hence by Lemma $2.3\{x \mid r(x) \neq 0\}-Q$, is finite. Hence $R-Q$, which $\subset\{x \mid r(x) \neq 0\}-Q$, is finite. Hence by Lemma $2.4 R$ has rank $\geqq m$. Therefore $f_{S}$ has rank $m$.

3. Functions that are not recursively approximable. It will now be shown that not every function is recursively approximable. That is to say, there are functions $f$ with the following property: there is an infinite nonrepeating recursive enumeration $x_{0}, x_{1}, \cdots$ such that, for every real number $E<1$ and every partial recursive function $\varphi$, $\varphi$ does not approximate $f$ with error $E$ on $x_{0}, x_{1}, \cdots$.

Let us call a function $f$ constructively nonrecursive if there is a recursive function $g$ such that, for all natural numbers $e, f(g(e)) \neq$ $\{e\}(g(e)) .^{10} \quad$ In view of Theorems 3.1 and 3.2 , the constructively nonrecursive functions form an uncountably infinite subclass of the functions that are not recursively approximable.

THEOREM 3.1. If a function is constructively nonrecursive, then it is not recursively approximable.

Proof. Let $f$ be any constructively nonrecursive function and $g$ a recursive function such that, for all $e$,

$$
f(g(e)) \neq\{e\}(g(e)) .
$$

First we will exhibit a recursive binary function $c$ such that, for all $i$ and $e$,

${ }^{10}$ For any $n \geqq 1$ and any $e, x_{1}, \cdots, x_{n}, "$ " $\{e\}\left(x_{1}, \cdots, x_{n}\right)$ " denotes the ambiguous value $\varphi\left(x_{1}, \cdots, x_{n}\right)$ of the partial recursive $n$-ary function $\varphi$ whose Gödel number is $e$. (Cf. [3], p. 340.) 


$$
\begin{gathered}
c(i, e)>i, \\
f(c(i, e)) \neq\{e\}(c(i, e)) .
\end{gathered}
$$

For this purpose, let $\psi$ be the partial recursive quaternary function defined by

$$
\psi(z, i, e, x) \simeq\left\{\begin{array}{l}
(z)_{x} \text { if } x \leqq i,{ }^{11} \\
\{e\}(x) \text { otherwise }
\end{array}\right.
$$

Then there is a primitive recursive ternary function $a$ such that

$$
\{a(z, i, e)\}(x) \simeq \psi(z, i, e, x) .^{12}
$$

Now for any natural numbers $i$ and $e$, let $z$ be the number $\Pi_{j \leqq i} p_{j}^{f(j)} \cdot{ }^{13}$ Because $a$ and $g$ are completely defined, $g(a(z, i, e))$ is defined. Hence either $g(a(z, i, e)) \leqq i$ or $g(a(z, i, e))>i$. But $g(a(z, i, e))$ cannot be $\leqq i$, for in that case

$$
\begin{aligned}
\{a(z, i, e)\}(g(a(z, i, e))) & \simeq[\operatorname{by}(3.5)] \psi(z, i, e, g(a(z, i, e,))) \\
& \simeq[b y(3.4)](z)_{g(a(z, i, e))} \simeq f(g(a(z, i, e))),
\end{aligned}
$$

contrary to (3.1). Hence $g(a(z, i, e))>i$. Therefore $\mu z(g(a(z, i, e))>i)$ is a recursive function of $i$ and $e$. It now follows that (3.6) and (3.7) define $b$ and $c$ as recursive binary functions.

$$
\begin{gathered}
b(i, e)=\mu z(g(a(z, i, e))>i), \\
c(i, e)=g(a(b(i, e), i, e)) .
\end{gathered}
$$

By (3.6) and (3,7), (3.2) holds. Now for any natural numbers $i$ and $e$, assume that $f(c(i, e))=\{e\}(c(i, e))$. Then $\{e\}(c(i, e))$ is defined and $f(g(a(b(i, e), i, e)))=[$ by $(3.7)] f(c(i, e))=\{e\}(c(i, e))=[$ by (3.2) and (3.4)] $\psi(b(i, e), i, e, c(i, e))=[$ by $(3.5)]\{a(b(i, e), i, e)\}(c(i, e))=[$ by $(3.7)]\{a(b(i, e)$, $i, e)\}(g(a(b(i, e), i, e)))$, contrary to (3.1). Therefore (3.3) follows by contradiction.

Next, define the primitive recursive functions $d$ and $e$ and the recursive function $x$ thus.

$$
\begin{gathered}
d(i)=\mu j((j+1) !>i) ; \\
e(i)=d(i)-(d(d(i))) ! ; \\
x(i)=\left\{\begin{array}{l}
0 \text { if } i=0, \\
c(x(i-1), e(i)) \text { otherwise. }
\end{array}\right.
\end{gathered}
$$

By (3.2), $x(0)<x(1)<\cdots$, so that $x(0), x(1), \cdots$ is an infinite nonrepeating recursive enumeration. We now show that, for any real

${ }^{11}$ For the notation $(z)_{x}$, cf. [3], p. 230.

12 Cf. [3], §65, Theorem XXIII.

13 For the notation $p_{j}$, cf. [3], p. 230. 
number $E<1$ and any partial recursive function $\varphi, \varphi$ does not approximate $f$ with error $E$ on $x(0), x(1), \cdots$, thereby proving that $f$ is not recursively approximable. The proof is by contradiction. Thus, assume that $\phi$ approximates $f$ with error $E$ on $x(0), x(1), \cdots$. Then there is a natural number $N$ such that

$$
\text { for all } n>N, \operatorname{err}(n) / n \leqq E .
$$

Choose any Gödel number $t$ of $\varphi$ and let $k$ be a natural number $>$ $\max (N, t, 1 /(1-E))$. Then for the $(k !+t+1) !-(k !+t)$ ! natural numbers $i$ such that $(k !+t) ! \leqq i<(k !+t+1) !, e(i)=t$; hence by (3.10) and (3.3) $f(x(i)) \neq\{t\}(x(i))$. Therefore $(k !+t+1) !>N$ and err $((k !+t+1) !) /(k !+t+1) ! \geqq 1-(k !+t) ! /(k !+t+1) !=1-1 /(k !+t+1)>E$, contrary to (3.11).

For Theorem 3.2, we use the following notation from [4]. For any natural number $e$, " $W_{e}$ " denotes the set of all numbers $y$ such that, for some $x,\{e\}(x)=y$. A set $P$ is productive if and only if there is a partial recursive function $\psi$ such that, for all $e$, if $W_{e} \subset P$ then $\psi(e) \in P-W_{e}$.

THEOREM 3.2. The representing function of any productive set is constructively nonrecursive. Hence uncountably many functions are not recursively approximable.

Proof. Given any productive set $P$, let $f$ be the function such that

$$
f(x)= \begin{cases}0 & \text { if } x \in P \\ 1 & \text { otherwise. }\end{cases}
$$

Myhill has shown that there is a recursive function $g$ such that, for all natural numbers $e$,

$$
g(e) \in\left(P-W_{e}\right) \cup\left(W_{e}-P\right) .^{14}
$$

Moreover there is a recursive function $h$ such that, for all natural numbers $e$,

$$
W_{h(e)}=\{y \mid\{e\}(y)=0\} .
$$

(For example, we can take for $h$ the primitive recursive function $\Lambda x \mu y(y \geqq x \&\{e\}(y)=0)$. For the $\Lambda$-notation, cf. [3], §65.) Now let $e$ be any natural number. By (3.12) $f(g(h(e)))=0$ if and only if $g(h(e)) \in P$; hence by (3.13) if and only if $g(h(e)) \notin W_{h(e)}$; hence by (3.14) if and only if $\{e\}(g(h(e))) \neq 0$. Thus $g(h)$ is a recursive function such that, for all $e, f(g(h(e))) \neq\{e\}(g(h(e)))$. Therefore $f$ is constructively 
nonrecursive.

It now follows from Theorem 3.1 that the representing functions of productive sets are not recursively approximable. Moreover, by [4], p. 47, there are uncountably many productive sets. Hence uncountably many functions are not recursively approximable.

REMARK. The proof of Theorem 3.2 can readily be generalized to show that a function $f$ is constructively nonrecursive if there is a recursively enumerable set $A$ and a productive set $P$ such that $f(x) \in A$ if and only if $x \in P$.

\section{BIBLIOGRAPHY}

1. M. Davis, Computability and unsolvability, New York: McGraw-Hill, 1958.

2. R. M. Friedberg, Three theorems on recursive enumeration. I. Decomposition. II. Maximal set. III. Enumeration without duplication, Journal of Symbolic Logic, 23 (1958), 309-316.

3. S. C. Kleene, Introduction to metamathematics, Amsterdam (North Holland), Groningen (Noordhoff), New York and Toronto (Van Nostrand), 1952.

4. H. Rogers, Jr., Theory of recursive functions and effective computability, Mimeographed by Massachusetts Institute of Technology, 1956, 1.

5. G. F. Rose, An extended notion of computability, International Congress for Logic, Methodology and Philosophy of Science (Stanford University, Stanford, California, August 24-September 2, 1960), Abstracts of contributed papers, p. 14.

System DeVelopment CoRporation

AND

UNIVERSITY OF CHICAGO 



\title{
PACIFIC JOURNAL OF MATHEMATICS
}

\author{
EDITORS
}

\author{
Ralph S. Phillips \\ Stanford University \\ Stanford, California \\ M. G. Arsove \\ University of Washington \\ Seattle 5 , Washington
}

\author{
J. DugunduI \\ University of Southern California \\ Los Angeles 7, California
}

Lowell J. Paige

University of California

Los Angeles 24, California

\section{ASSOCIATE EDITORS}

E. F. BECKENBACH

T. M. CHERRY
D. DERRY
M. OHTSUKA

H. L. ROYDEN

E. SPANIER
E. G. STRAUS

F. WOLF

\section{SUPPORTING INSTITUTIONS}

UNIVERSITY OF BRITISH COLUMBIA CALIFORNIA INSTITUTE OF TECHNOLOGY UNIVERSITY OF CALIFORNIA MONTANA STATE UNIVERSITY UNIVERSITY OF NEVADA NEW MEXICO STATE UNIVERSITY OREGON STATE UNIVERSITY UNIVERSITY OF OREGON OSAKA UNIVERSITY UNIVERSITY OF SOUTHERN CALIFORNIA
STANFORD UNIVERSITY

UNIVERSITY OF TOKYO

UNIVERSITY OF UTAH

WASHINGTON STATE UNIVERSITY

UNIVERSITY OF WASHINGTON

AMERICAN MATHEMATICAL SOCIETY CALIFORNIA RESEARCH CORPORATION SPACE TECHNOLOGY LABORATORIES NAVAL ORDNANCE TEST STATION

Mathematical papers intended for publication in the Pacific Journal of Mathematics should be typewritten (double spaced), and the author should keep a complete copy. Manuscripts may be sent to any one of the four editors. All other communications to the editors should be addressed to the managing editor, L. J. Paige at the University of California, Los Angeles 24, California.

50 reprints per author of each article are furnished free of charge; additional copies may be obtained at cost in multiples of 50 .

The Pacific Journal of Mathematics is published quarterly, in March, June, September, and December. Effective with Volume 13 the price per volume (4 numbers) is $\$ 18.00$; single issues, $\$ 5.00$. Special price for current issues to individual faculty members of supporting institutions and to individual members of the American Mathematical Society: $\$ 8.00$ per volume; single issues \$2.50. Back numbers are available.

Subscriptions, orders for back numbers, and changes of address should be sent to Pacific Journal of Mathematics, 103 Highland Boulevard, Berkeley 8, California.

Printed at Kokusai Bunken Insatsusha (International Academic Printing Co., Ltd.), No. 6, 2-chome, Fujimi-cho, Chiyoda-ku, Tokyo, Japan.

PUBLISHED BY PACIFIC JOURNAL OF MATHEMATICS, A NON-PROFIT CORPORATION

The Supporting Institutions listed above contribute to the cost of publication of this Journal, but they are not owners or publishers and have no responsibility for its content or policies. 


\section{Pacific Journal of Mathematics}

\section{Vol. 13, No. 2 \\ April, 1963}

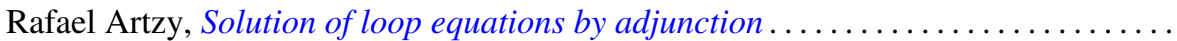

Earl Robert Berkson, A characterization of scalar type operators on reflexive

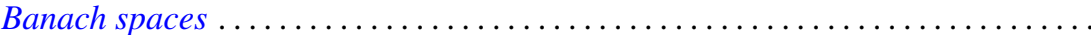

Mario Borelli, Divisorial varieties

365

Raj Chandra Bose, Strongly regular graphs, partial geometries and partially

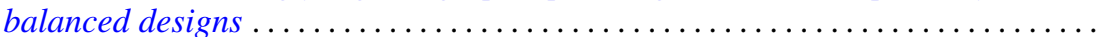

389

R. H. Bruck, Finite nets. II. Uniqueness and imbedding ............... 421

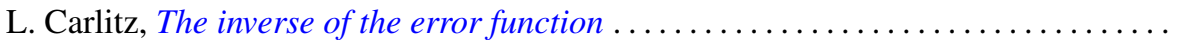

Robert Wayne Carroll, Some degenerate Cauchy problems with operator

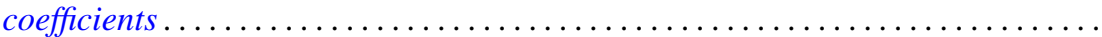

Michael P. Drazin and Emilie Virginia Haynsworth, A theorem on matrices of 0 's

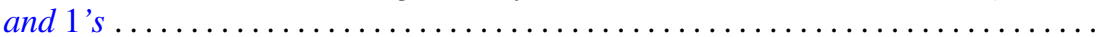

Lawrence Carl Eggan and Eugene A. Maier, On complex approximation .......... James Michael Gardner Fell, Weak containment and Kronecker products of group

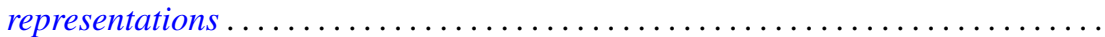

Paul Chase Fife, Schauder estimates under incomplete Hölder continuity

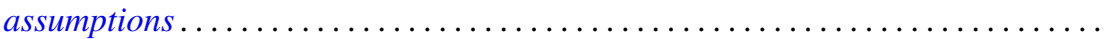

Shaul Foguel, Powers of a contraction in Hilbert space ...................

Neal Eugene Foland, The structure of the orbits and their limit sets in continuous

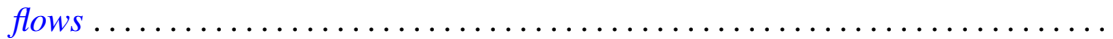

Frank John Forelli, Jr., Analytic measures . . . . . . . . . . . . . . . . . . . . . 563

Robert William Gilmer, Jr., On a classical theorem of Noether in ideal theory ....... P. R. Halmos and Jack E. McLaughlin, Partial isometries .

Albert Emerson Hurd, Maximum modulus algebras and local approximation in

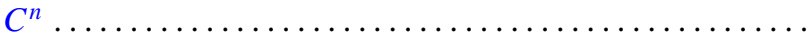

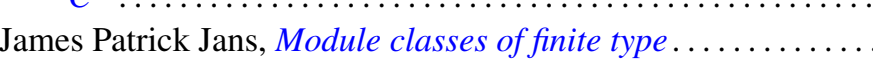

Betty Kvarda, On densities of sets of lattice points ...

H. Larcher, A geometric characterization for a class of discontinuous groups of linear fractional transformations .

John W. Moon and Leo Moser, Simple paths on polyhedra .

T. S. Motzkin and Ernst Gabor Straus, Representation of a point of a set as sum of

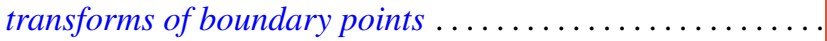

Rajakularaman Ponnuswami Pakshirajan, An analogue of Kolmogorov's three-series theorem for abstract random variables ...............

Robert Ralph Phelps, Čebyšev subspaces of finite codimension in $C(X)$...

James Dolan Reid, On subgroups of an Abelian group maximal disjoint from a given subgroup ...

William T. Reid, Riccati matrix differential equations and non-oscillation criteria for associated linear differential systems .................

Georg Johann Rieger, Some theorems on prime ideals in algebraic number fields ...

Gene Fuerst Rose and Joseph Silbert Ullian, Approximations of functions on the

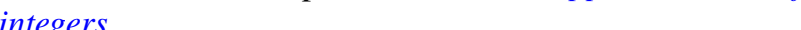

F. J. Sansone, Combinatorial functions and regressive isols . . . . . . . . . . 703

Leo Sario, On locally meromorphic functions with single-valued moduli . . . . . . . 709

Takayuki Tamura, Semigroups and their subsemigroup lattices.

Pui-kei Wong, Existence and asymptotic behavior of proper solutions of a class of second-order nonlinear differential equations . . ........... 